\title{
From pharmacogenetics to imaging pharmacogenetics: elucidating mechanisms of antidepressant response
}

\author{
“...neuroimaging intermediate phenotypes with analyses of \\ pharmacogenetic variants bears promise for an improved \\ understanding of modulation of antidepressant effects by \\ genetic variation..."
}

First draft submitted: 10 May 2017; Accepted for publication: 12 May 2017; Published online: 22 June 2017

Keywords: 5 -HTTLPR $\bullet$ antidepressant response $\bullet$ imaging $\bullet$ pharmacogenetics

Psychiatric pharmacogenetics research has helped to elucidate biological mechanisms of response to a number of medications. However, compared with other medical areas, such as cancer treatment, clinical applicability of these findings is still limited by a number of shortcomings of currently available data. We discuss the potential advantages of combining imaging and pharmacogenetics approaches to better describe the underlying mechanisms of antidepressant response and how this approach may be applied to future studies.

Medication with antidepressants is a cornerstone in treatment of major depression and other major psychiatric disorders. High rates of nonresponse and side effects contribute to an unsatisfactory outcome for many patients. Therefore, there has been considerable effort to identify clinical and biological predictors of antidepressant response, and numerous studies on psychiatric pharmacogenetics have been undertaken over the past decades. However, meta-analytic evidence for an influence on antidepressant response only exists for a limited number of genetic variants, which we will briefly summarize.

The 5-HTTLPR insertion-deletion polymorphism in the promoter region of the serotonin transporter gene has been subject of numerous studies. Carriers of the short (s-) allele, which is linked to altered serotonin turnover, of European descent showed poorer response, especially to selective serotonin reuptake inhibitors (SSRI), in several meta-analyses (e.g., [1]). Of note, the G-allele of the rs25531 polymorphism leads to an expression of serotonin in long (l-) allele carriers that is functionally equivalent to s-allele carriers; however, not all studies have incorporated rs25531 in their analyses which may contribute to some inconsistent results.

Of the genes in the hypothalamicpituitary-adrenal axis, FKBP5, encoding a co-chaperon protein involved in the affinity of glucocorticoid receptors for cortisol, is the most promising candidate for antidepressant response. Although the function of most polymorphisms has not fully been elucidated yet, meta-analytic evidence exists for several variants, including rs4713916 [2], rs3800373 and rs1360780 [3].

Extensive research has also been performed on rs6265 (Val66Met) in the BDNF gene. The Met-allele is linked to lower activity-dependent BDNF secretion. Despite mixed findings, recent meta-analyses indicated better treatment response, especially to SSRI, in heterozygous Met/Val individuals, particularly of Asian descent [3].

CYP2D6 and CYP2C19 have been the focus of research in pharmacokinetics of antidepressants. A combination of haplotypes and copy number variants leads to large differences in metabolic activity, ranging from no (poor metabolizer) to reduced (intermediate metabolizer), normal (extensive metabolizer) to increased activity (ultrarapid metabolizer).

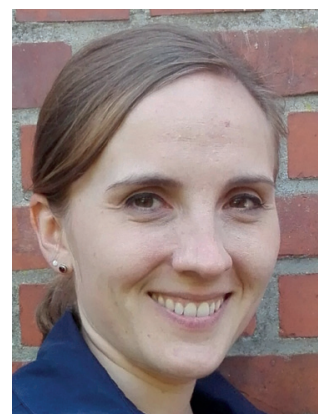

Eva J Brandl

Author for correspondence: Department of Psychiatry \& Psychotherapy, Campus Mitte, Charité Universitätsmedizin Berlin, Berlin, Germany

and

Berlin Institute of Health (BIH), Berlin, Germany

Tel.: +493023112120

Fax: +49 3023112790

eva.brandl@charite.de

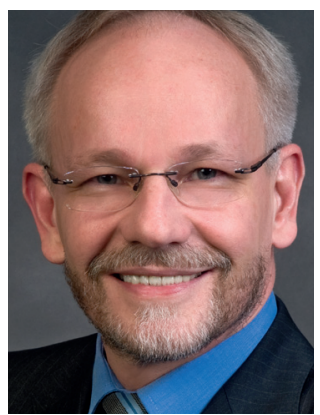

Henrik Walter

Department of Psychiatry \& Psychotherapy, Campus Mitte, Charité Universitätsmedizin Berlin, Berlin, Germany

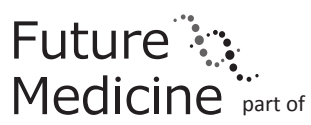
fsg 
However, the activity of the enzymes is highly influenced by environmental factors such as diet, age and medication. This interaction has not been considered sufficiently in many studies. There is also no clear standard for genotyping and sequencing of cytochrome genes across studies [4]. Together, these factors may explain the highly mixed findings for these enzymes. While there is a known impact of genetic variation on plasma concentrations of antidepressants, association with treatment response is less clear. Nonetheless, several studies indicate a higher risk for nonresponse in ultrarapid metabolizer and for side effects in poor metabolizer, leading to first clinical recommendations for dose adjustments in patients with nonextensive metabolizer genotypes (e.g., [5]).

Polymorphisms in the $A B C B 1$ gene, encoding a transport protein that is involved in the passage of the blood-brain barrier of most antidepressants, have also been associated with antidepressant response, although their functional relevance remains to be further investigated. Meta-analytic associations with antidepressant response have been reported for rs2032582 and rs2032583 [3,6].

A number of other candidate genes for antidepressant response have yielded interesting, however mixed, findings, among these genes encoding guanine nucleotide binding protein beta polypeptide 3 , tryptophan hydroxylase, ionotropic glutamate kainate 4 receptor KA1 and several inflammatory genes [7].

In general, available data from candidate gene studies on antidepressant response are limited by small-tomoderate effect sizes of the variants, limited sample sizes, mixed study medications, lacking consideration of gene-environment effects and other confounders. Moreover, epigenetic modifications might be important modifiers of antidepressant response, but have only been investigated in few studies to date.

"

"...changes in amygdala activity in response to negative stimuli during antidepressant treatment occur ... indicating a differential effect of the medication depending on genotype."

Another issue is the lacking association of any of the candidate genes with treatment response in genomewide association studies (GWAS). A meta-analysis of three large GWAS on antidepressant response, the Sequenced Treatment Alternatives to Relieve Depression, the Genome-based Therapeutic Drugs for Depression and the Munich Antidepressant Response Signature studies, yielded no finding with genomewide significance $[8]$. Also in other large GWAS on antidepressant response such as the International SSRI Pharmacogenomics Consortium [9], no results with genome-wide significance were obtained.
Despite these limitations, several variants have been incorporated in first commercial pharmacogenetic tests for antidepressant response. However, general recommendations for application of these tests in clinical practice cannot be made based on currently available data [10]. Genetic testing for cytochrome variants may be useful in patients with nonresponse, whose plasma levels differ significantly from what would be expected at a certain dosage after excluding other reasons for the differences. Nonetheless, cost-effectiveness and improvement of outcome based on pharmacogenetic testing are not yet supported sufficiently by the literature [11]. On the other hand, it has been postulated that the cost-effectiveness of one analysis for a specific gene-drug pair may not be the ideal approach, considering genotyping is only necessary once for each individual and that it is likely that the information will be relevant for other medications as well [12].

One way of achieving a better understanding of genetic influences on antidepressant response may be the combination of brain imaging with pharmacogenetics as structural and functional alterations in the brain have been linked to pathogenesis of depression as well as to antidepressant response. MRI studies have identified several potential imaging markers for antidepressant response. For example, normalization of amygdala hypoactivity during the face-processing task in responders, but not in nonresponders [13] has been reported. Activation during the go/no-go task in the inferior frontal gyrus, the amygdala, insula and the anterior cingulate cortex at baseline of antidepressant treatment has been correlated with reduction of depression symptoms during the course of treatment [14]. Resting-state fMRI studies have delivered a number of associations with antidepressant response, among these are increased functional connectivity between frontal and limbic regions, connectivity of visual circuits and hyperconnectivity of the default mode network [15]. However, similar to most pharmacogenetics studies, small sample sizes and lacking standardization in terms of design, applied paradigms and medications limit the understanding of imaging correlates of antidepressant response to date.

To date, there is a limited number of studies combining both pharmacogenetics and imaging techniques, and even less studies including longitudinal response measures. Most of these studies have examined the 5-HTTLPR polymorphism. Available data show that changes in amygdala activity in response to negative stimuli during antidepressant treatment occur rather in l-allele homozygotes than in s-allele carriers (e.g., [16]), indicating a differential effect of the medication depending on genotype. Other studies have found altered brain activation in response to happy faces and 
nonremission to antidepressant treatment in carriers of the G-allele of the rs1049353 polymorphism in the cannabinoid receptor 1 gene [17], as well as an influence of variants in $B D N F, I L 1 B$ and in $N P Y$ [7]. More recently, changes in levels of microRNAs, which are involved in regulation of gene expression, have been associated with changes in brain connectivity and activity in response to antidepressant treatment [18]. However, the number of studies applying longitudinal assessments of imaging phenotypes as well as of response is extremely small to date. Considering the functional implication of CYP2D6 in the brain [19] as well as the potential impact of CYP2D6 and other pharmacokinetic enzymes on drug concentrations in the brain, imaging-pharmacogenetics studies on variation in pharmacokinetic genes and antidepressant response would be of special interest since they would elucidate these potentially complex effects of variation in enzymes and transporters [20]; however, to date there are no published studies examining these variants and their impact on antidepressant outcome in imaging studies.

Shortcomings of available imaging-pharmacogenetics studies are not only their limited number, but also the small sample sizes, lacking standardization of imaging paradigms and the added cost to research [21]. Despite these issues, the combination of neuroimaging intermediate phenotypes with analyses of pharmacogenetic variants bears promise for an improved understanding of modulation of antidepressant effects by genetic variation as well as for future

\section{References}

1 Porcelli S, Fabbri C, Serretti A. Meta-analysis of serotonin transporter gene promoter polymorphism (5-HTTLPR) association with antidepressant efficacy. Eur. Neuropsychopharmacol. 22(4), 239-258 (2012).

2 Zou YF, Wang F, Feng XL et al. Meta-analysis of FKBP5 gene polymorphisms association with treatment response in patients with mood disorders. Neurosci. Lett. 484(1), 56-61 (2010).

3 Niitsu T, Fabbri C, Bentini F, Serretti A. Pharmacogenetics in major depression: a comprehensive meta-analysis. Prog. Neuropsychopharmacol. Biol. Psychiatry 45, 183-194 (2013).

4 Kalman LV, Agundez J, Appell ML et al. Pharmacogenetic allele nomenclature: international workgroup recommendations for test result reporting. Clin. Pharmacol. Ther. 99(2), 172-185 (2016).

5 Hicks JK, Bishop JR, Sangkuhl K et al. Clinical Pharmacogenetics Implementation Consortium (CPIC) guideline for CYP2D6 and CYP2C19 genotypes and dosing of selective serotonin reuptake inhibitors. Clin. Pharmacol. Ther. 98(2), 127-134 (2015). treatment options through identification of biomarkers for response, identification of earlier response predictors before clinical changes in symptom severity occur, and supporting development of novel substances. Future studies in this field should apply standardized paradigms in larger, well-characterized samples of patients receiving antidepressant treatment with longitudinal outcome measures. Furthermore, using more advanced neuroimaging modalities such as high angular resolution diffusion imaging or neurite orientation dispersion and density imaging [22] may help to better delineate potential predictors of or markers of neuroplasticity associated with treatment response. Ongoing large trials such as the Canadian biomarker integration network in depression [23] will be the first step toward a deeper understanding of mechanisms of antidepressant response, making imaging-pharmacogenetics one of the most promising strategies in research on factors influencing antidepressant response to date.

\section{Financial \& competing interests disclosure}

$\mathrm{H}$ Walter has received speaker fees from Servier. EJ Brandl is a participant in the BIH Charité Clinician Scientist Program funded by Charité - Universitätsmedizin Berlin and the Berlin Institute of Health. The authors have no other relevant affiliations or financial involvement with any organization or entity with a financial interest in or financial conflict with the subject matter or materials discussed in the manuscript apart from those disclosed.

No writing assistance was utilized in the production of this manuscript.
6 Breitenstein B, Bruckl TM, Ising M, Muller-Myhsok B, Holsboer F, Czamara D. $A B C B 1$ gene variants and antidepressant treatment outcome: a meta-analysis. Am. J. Med. Genet. B Neuropsychiatr. Genet. 168B(4), 274-283 (2015).

7 Lett TA, Walter H, Brandl EJ. Pharmacogenetics and imaging-pharmacogenetics of antidepressant response: towards translational strategies. CNS Drugs 30 (12), 1169-1189 (2016).

8 GENDEP Investigators, MARS Investigators, STAR*D Investigators. Common genetic variation and antidepressant efficacy in major depressive disorder: a meta-analysis of three genome-wide pharmacogenetic studies. Am. J. Psychiatry 170(2), 207-217 (2013).

9 Biernacka JM, Sangkuhl K, Jenkins G et al. The International SSRI Pharmacogenomics Consortium (ISPC): a genome-wide association study of antidepressant treatment response. Transl. Psychiatry 5, e553 (2015).

10 Bschor T, Baethge C, Hiemke C, Muller-Oerlinghausen B. [Genetic tests for controlling treatment with antidepressants]. Nervenarzt 88(5), 495-499 (2017).

11 Rosenblat JD, Lee Y, Mcintyre RS. Does pharmacogenomic testing improve clinical outcomes for major depressive 
disorder? A systematic review of clinical trials and costeffectiveness studies. J. Clin. Psychiatry 78(6), 720-729 (2017).

12 Leeder JS, Gaedigk A. CYP2D6 and pharmacogenomics: where does future research need to focus? Part 2: clinical aspects. Pharmacogenomics 15(8), 1055-1058 (2014).

13 Williams LM, Korgaonkar MS, Song YC et al. Amygdala reactivity to emotional faces in the prediction of general and medication-specific responses to antidepressant treatment in the randomized iSPOT-D trial. Neuropsychopharmacology 40(10), 2398-2408 (2015).

14 Langenecker SA, Kennedy SE, Guidotti LM et al. Frontal and limbic activation during inhibitory control predicts treatment response in major depressive disorder. Biol. Psychiatry 62(11), 1272-1280 (2007).

15 Dichter GS, Gibbs D, Smoski MJ. A systematic review of relations between resting-state functional-MRI and treatment response in major depressive disorder. J. Affect Disord. 172, 8-17 (2015).

16 Ramasubbu R, Burgess A, Gaxiola-Valdez I et al. Amygdala responses to quetiapine $\mathrm{XR}$ and citalopram treatment in major depression: the role of 5-HTTLPR-S/ Lg polymorphisms. Hum. Psychopharmacol. 31(2), 144-155 (2016).

17 Domschke K, Dannlowski U, Ohrmann P et al. Cannabinoid receptor 1 (CNRI) gene: impact on antidepressant treatment response and emotion processing in major depression. Eur. Neuropsychopharmacol. 18(10), 751-759 (2008).

18 Lopez JP, Pereira F, Richard-Devantoy $S$ et al. Co-variation of peripheral levels of miR-1202 and brain activity and connectivity during antidepressant treatment. Neuropsychopharmacology doi:10.1038/npp.2017.9 (2017) (Epub ahead of print).

19 Stingl JC, Esslinger C, Tost $\mathrm{H}$ et al. Genetic variation in CYP2D6 impacts neural activation during cognitive tasks in humans. Neuroimage 59(3), 2818-2823 (2012).

20 Viviani R, Lehmann ML, Stingl JC. Use of magnetic resonance imaging in pharmacogenomics. Br. J. Clin. Pharmacol. 77(4), 684-694 (2014).

21 Falcone M, Smith RM, Chenoweth MJ et al. Neuroimaging in psychiatric pharmacogenetics research: the promise and pitfalls. Neuropsychopharmacology 38(12), 2327-2337 (2013).

22 Zhang H, Schneider T, Wheeler-Kingshott CA, Alexander DC. NODDI: practical in vivo neurite orientation dispersion and density imaging of the human brain. Neuroimage 61(4), 1000-1016 (2012).

23 Lam RW, Milev R, Rotzinger S et al. Discovering biomarkers for antidepressant response: protocol from the Canadian biomarker integration network in depression (CAN-BIND) and clinical characteristics of the first patient cohort. $B M C$ Psychiatry 16, 105 (2016). 